\title{
Cyclic changes in the vaginal epithelium of normal rhesus macaques
}

\author{
B Poonia, L Walter, J Dufour ${ }^{1}$, R Harrison', P A Marx ${ }^{2}$ and R S Veazey \\ Division of Comparative Pathology, Tulane National Primate Research Centre, 18703 Three Rivers Road, Covington, Louisiana 70433, USA \\ ${ }^{1}$ Division of Veterinary Medicine, Tulane National Primate Research Centre, 18703 Three Rivers Road, Covington, Louisiana 70433, USA \\ ${ }^{2}$ Division of Microbiology, Tulane National Primate Research Center, 18703 Three Rivers Road, Covington, Louisiana 70433, USA \\ (Requests for offprints should be addressed to R S Veazey; Email: rveazey@tulane.edu)
}

\begin{abstract}
Studies in nonhuman primates indicate that changes in the thickness and integrity of the vaginal epithelium affect the transmission rates of HIV-1, but few studies have examined the normal variations that may occur in the vagina of normal macaques as a result of aging or changes in the menstrual cycle. This study was conducted to determine if differences occur in the thickness of the vaginal mucosa with age or menses. Vaginal mucosal thickness was compared in 46 rhesus macaques grouped as juvenile (1-3 years old), mature cycling (3-21 years old), and geriatric ( $>21$ years old). Epithelia of mature cycling macaques were also compared at different stages of the menstrual cycle. Older females ( $>21$ years) had the thinnest and least keratinized epithelium of all groups,
\end{abstract}

followed by the youngest females ( $<3$ years). The vaginal epithelium was also thinner in cycling macaques during menses compared to the follicular stage. In addition, young, geriatric, or cycling macaques during menses had minimal keratinization. We hypothesize that normal physiologic changes in the vaginal epithelium of women occur with age and menses, which may affect a woman's susceptibility to HIV-1 transmission and other sexually transmitted diseases. Also, age and menstrual cycle should be considered when designing vaginal transmission experiments in rhesus macaques.

Journal of Endocrinology (2006) 190, 829-835

\section{Introduction}

Worldwide, over $80 \%$ of all HIV-1 transmissions occur as a result of vaginal (heterosexual) exposure, and women account for approximately half of the 40 million persons presently living with HIV-1 infection (Quinn et al. 2000, Shattock \& Moore 2003). Although the female reproductive tract is obviously the major site of HIV-1 transmission, the exact sites and mechanisms involved in early viral entry and dissemination in women are largely unknown and presently debated. For example, it is not known with certainty whether or not the majority of transmissions occur across an intact vagina, cervix, or even the endometrium following exposure to HIV-1-infected semen. However, rhesus macaques that have had their uterus and cervix surgically removed remain susceptible to transmission following vaginal exposure indicating that the vaginal mucosa alone is sufficient for transmission (Miller et al. 1992).

Early studies of simian immunodeficiency virus (SIV) vaginal transmission demonstrated that 100 to $>1000$ times more virus was required to establish the infection of macaques through an intravaginal inoculation compared with an i.v. inoculation (Miller et al. 1989, 1992, Marx et al. 1993, Sodora et al. 1998). However, even when large doses of virus are 'randomly' applied (with no consideration to age or the menstrual cycle) to the vagina of macaques, only a fraction of animals become infected. Therefore, large numbers of animals are usually necessary to demonstrate statistical significance in studies examining rates of vaginal transmission or protection.

Previous studies have demonstrated that macaques are more susceptible to vaginal transmission during menses (Sodora et al. 1998). However, only one comparative study of the vaginal mucosal thickness and integrity at different stages of the menstrual cycle has been performed in macaques, which showed that the number and distribution of immune cells remained constant in the vagina, despite changes in the epithelial thickness (Ma et al. 2001). We hypothesized that normal cyclic changes in the vaginal thickness and integrity occur during the follicular (estrogen phase) and luteal (menstrual phase) of normal adult cycling macaques. Moreover, we hypothesized that the vaginal epithelium of macaques of different ages (prepubescent juveniles or geriatric 'postmenopausal' macaques) would be thinner than those from normal cycling adult female macaques.

In this paper, we describe the normal histologic changes in the thickness and integrity of the vaginal epithelium that occur due to age or changes in the menstrual cycle of rhesus macaques. These data demonstrate that significant cyclic 
changes in the vaginal mucosa occur with age and menses, which may affect vaginal SIV transmission rates, and by inference, vaginal HIV-1 transmission in humans. Moreover, these data suggest that using rhesus macaques at different stages of the menstrual cycle or those of different age groups could significantly impact the results or interpretation of prevention, vaccine, or other vaginal transmission studies.

\section{Materials and Methods}

\section{Assessment of changes in vaginal epithelial thickness due to age}

Vaginal mucosal tissues were collected and examined from 46 female rhesus macaques (Macacca mulatta) at necropsy over a 5-year period at the Tulane National Primate Research Center (TNPRC). These animals were all born and housed in outdoor breeding corrals at the TNPRC. All monkeys were maintained in accordance with the standards of the American Association for Accreditation of Laboratory Animal Care, and all studies were approved by the Tulane Institutional Animal Care and Use Committee. None of the macaques were infected with SIV; most were humanely euthanized as controls for tissue collection on various research projects, or due to non-reproductive-related illnesses, including colitis, trauma, or seropositivity to simian retrovirus type D. All tissues were fixed in 10\% neutral buffered formalin, paraffin embedded, and sectioned and stained with hematoxylin and eosin ( $\mathrm{H} \& \mathrm{E})$ using routine procedures. The macaques were categorized into three age groups before analysis: (1) young prepubescent juveniles 1-3 years of age, (2) mature cycling females 3-21 years of age, and (3) older geriatric females $>21$ years of age. Serum for hormone profiles of these macaques was not available for this part of the study.

\section{Assessment of changes in cycling macaques at different stages of the menstrual cycle}

To evaluate the changes in vaginal epithelium during the normal menstrual cycle, vaginal epithelium from six normal, healthy, cycling female rhesus macaques between $5 \cdot 8$ and 10 years of age were examined. These animals were also born and raised in outdoor breeding corrals at the TNPRC until they were assigned to this study, at which time they were transferred indoors and individually cage-housed in a biosafety level 2 research facilities in accordance with the standards of the American Association for Accreditation of Laboratory Animal Care. Animals were acclimated to their cages for 3-6 months before testing procedures began. To assess the stage of the menstrual cycle, macaques were first placed on a menses watch (observations began in mid-June) in which they were observed daily to detect the first day of vaginal blood, signaling the onset of menses (day 0 of the menstrual cycle). Since the average cycle in macaques is 28 days, the vaginal mucosa was biopsied exactly 14 days later and these samples were used to represent 'follicular' stage biopsies. From day 0, the vaginal mucosa was again biopsied exactly 28 days later for luteal-phase samples. Beginning on day 14 (peak follicular phase), macaques were briefly sedated with $10 \mathrm{mg} / \mathrm{kg}$ i.m. ketamine (Fort Dodge Animal Health, Fort Dodge, IA, USA) and sera was collected every third day for 35 days subsequently to assess hormone profiles and confirm these cyclic stages by estrogen and progesterone levels (see below). In all cases, vaginal biopsies were collected from macaques anesthetized with Telazol $(8 \mathrm{mg} / \mathrm{kg}$ i.m.; Fort Dodge Animal Health) using $3 \mathrm{~mm}$ biopsy forceps. For these procedures, anesthetized macaques were placed in modified ventral recumbency with their hips slightly elevated and ventral a full thickness mucosal biopsy of vaginal epithelium that included the surface to the basal epithelial layer was collected from the lateral vaginal wall. All studies and procedures were approved by the Tulane Institutional Animal Care and Use Committee.

Sections of the vaginal epithelium were prepared as above and vaginal epithelial thickness in both biopsy and necropsy samples was measured using ImageProPlus software, version 4.5 (Media Cybernetics, Silver Springs, MD, USA) on appropriately oriented $\mathrm{H} \& \mathrm{E}$ stained sections using a Leica microscope (Leica Microsystems Inc., Bannockbum, IL, USA) interfaced to a digital camera (Spot Insight color camera; Diagnostic Instruments Inc., Sterling Heights, MI, USA). The software was calibrated for each objective using a stage micrometer and all measurements were performed at $200 \times$ magnification. For each section, 15 different lengths were measured by applying an electronic grid in the ImageProPlus software and efforts were made to minimize bias by measuring at every other grid line for all the tissues. The 15 thickness measurements were used to calculate the average thickness for that particular animal. Since vaginal tissues varied in thickness in the same sections due to the presence of undulating rete pegs (peg-like extensions of the epithelium into the underlying lamina propria), we also measured the epithelium at the thinnest point in the section to assess the 'weakest link' in the squamous epithelium.

\section{Hormone levels}

Levels of serum progesterone and estrogen were examined from the seven macaques described above using a commercial CoatA-Count kit (Diagnostic Products Co., Los Angeles, CA, USA). The assay is a solid-phase competitive RIA, wherein known amounts of ${ }^{125}$ I-labeled hormone competes for a fixed time with the hormone in the serum sample for antibody sites bound on the tubes. Tubes were then decanted and analyzed in a gamma counter (Packard Instrument Co., Meriden, CT, USA), which yields a value that is then compared to a standard curve. For the estradiol assay, only estrone (10\%) and D-equilenin (4 - 4\%) show any cross-activity above $1.0 \%$. For the progesterone assay, the only cross-activity above $1.0 \%$ is 11 -deoxy-corticosterone $(2 \cdot 2 \%)$, hydroprogesterone $(3 \cdot 4 \%), 5$ a-pregnan-3,20-dione $(9 \cdot 0 \%)$, and 5 b-pregnan-3,20-dione $(3 \cdot 2 \%)$. 


\section{Statistical analysis}

Differences between the groups were analyzed by paired Student's $t$-test, means \pm S.D. are shown. Differences with a $P$ value $<0 \cdot 05$ were considered significant.

\section{Results}

\section{Effect of age on vaginal epithelial thickness}

There were clear and highly significant differences in the vaginal epithelium of female rhesus macaques due to age (Figs 1 and 2). The average vaginal epithelial thickness increased as the macaque matured from non-cycling juveniles to mature, cycling females. The epithelium remained thick until about 20 years of age, after which the thickness was significantly reduced. The epithelial thickness was significantly higher in 3-21-year-old age group $(P<$ $0 \cdot 01)$ when compared with either the juveniles $(<3$ years old) or the older females ( $>21$ years old). The older females had the thinnest vaginal epithelium of all the four groups (Fig. 1). The epithelium in $>21$ years age group was significantly thinner when compared with both $<3$ years $(P<0 \cdot 03)$ and $3-21$ years $(P<0 \cdot 01)$ age groups. The thinnest part of the vaginal epithelium in each section was also determined in order to see the age-related changes in that area. Interestingly, there was not much difference in the thinnest part of epithelium among the different age groups (Fig. 1).

\section{Effect of menstrual cycle on vaginal epithelium}

The epithelial thickness was measured in six female rhesus macaques under menses watch.

In this study, the first day of the menstrual cycle was estimated based on the first day of menses (vaginal blood discharge) observed. Vaginal biopsies were collected 14 and 28 days later using the assumption that each animal had a 28-day cycle. To confirm this, serum was collected and retrospectively analyzed for both estrogen and progesterone levels on these time-points, and at 3-day intervals throughout a 35-day period encompassing the dates when the biopsies were collected. Figure $3 \mathrm{~A}$ demonstrates that the peak estrogen phase was actually closer to 11 days after the first day of menses rather than at day 14 when the biopsies were collected but regardless, the data show that the biopsies were collected soon after the estrogen peak. The biopsies collected at day 28 (luteal phase) were also confirmed to have been taken at a time of progesterone dominance (low estrogen levels, high progesterone levels; Fig. 3A). The vaginal epithelial thickness

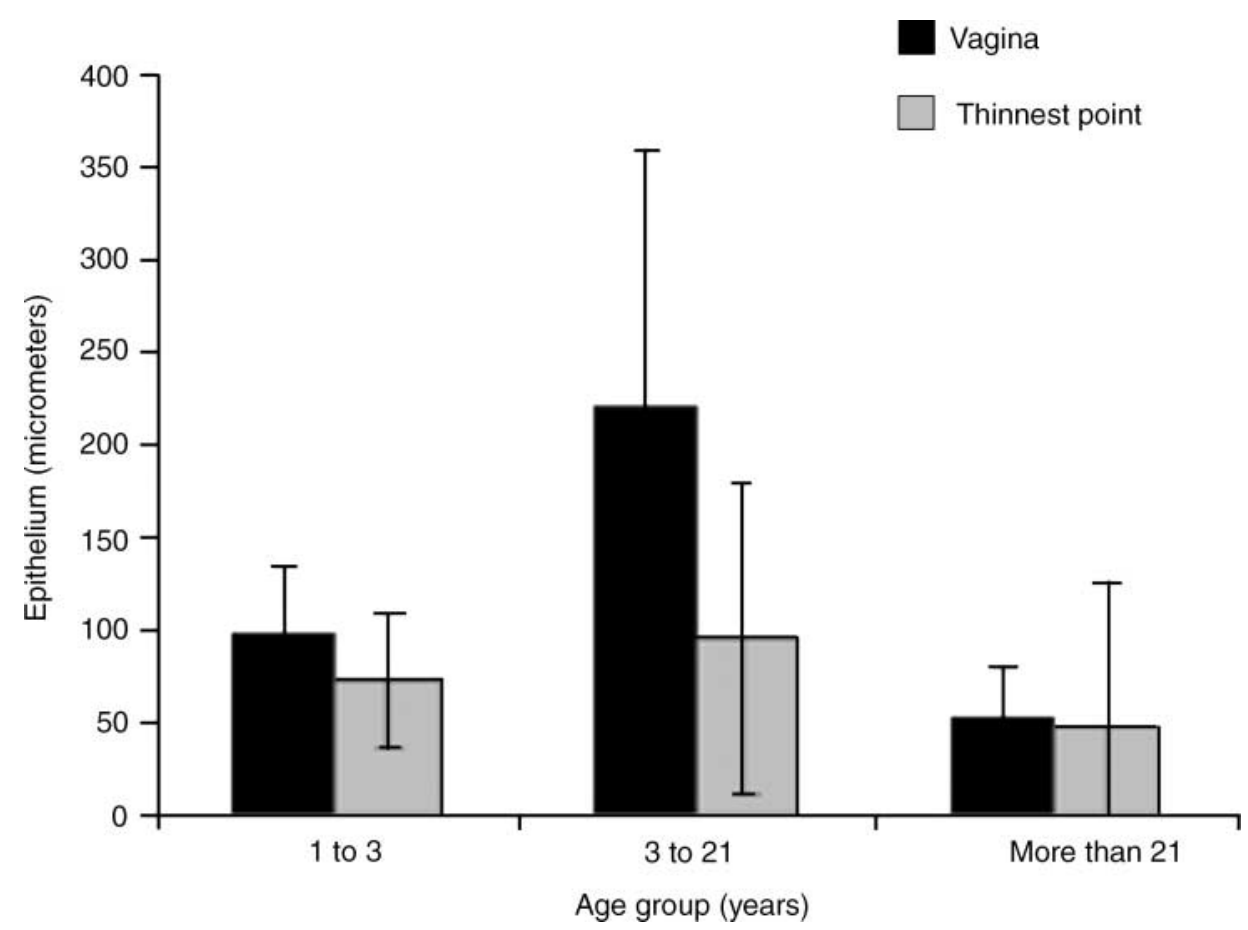

Figure 1 Thickness of vaginal epithelium (in micrometers) in macaques of different ages. Sections from vaginal wall (black bars) as well as thinnest point of the section (gray bars) were examined and measured. Values represent means of 8 (1-3 years), 42 (3-21 years), or 5 (more than 21 years) animals. Both young $(<3$ years) and aged ( $>21$ years) macaques had significantly $(P<0 \cdot 01)$ thinner epithelia compared with 3-21-year-old macaques. Aged macaques had significantly thinner epithelium when compared with $<3$-year-old macaques $(P<0 \cdot 03)$. Means \pm s.D. 

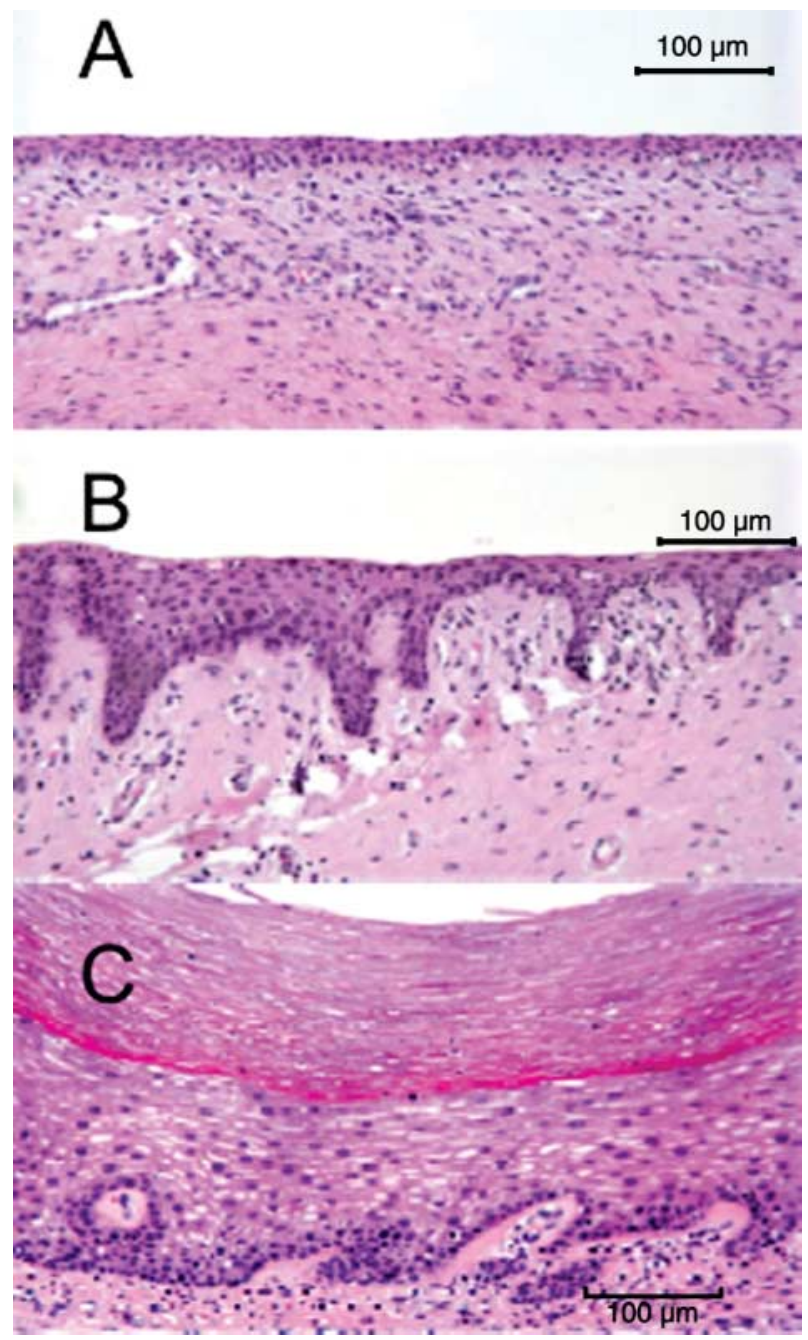

Figure 2 Representative photomicrographs of vaginal epithelium from macaques of different age groups show that (A) very young (1 year old) or (B) geriatric (30 years old) females have significantly thinner epithelia compared with (C) mature cycling females (10 years old). Magnification X 200.

decreased during the luteal phase of the menstrual cycle in all macaques, at which time the epithelium was significantly $(P<0 \cdot 01)$ thinner than during follicular stage (Fig. 3B). In addition, there was marked keratinization of the superficial layers of the epithelium during the follicular phase, which was essentially absent during peak luteal phase (Fig. 4).

\section{Discussion}

In the present study, we show that there are significant alterations in the vaginal epithelial thickness and integrity of female rhesus macaques that occur both as the result of aging as well as in different stages of the menstrual cycle of cycling macaques. We have previously shown that changes in the thickness in the vaginal mucosa can markedly affect transmission rates of SIV in macaques (Marx et al. 1996, Veazey et al. 2003a), but to our knowledge, few studies have examined normal cyclic changes in the vaginal epithelium of macaques of different ages, or at different stages of the menstrual cycle. If such dynamic changes also occur in the vaginal epithelium of women, it is likely that such changes in the integrity of this mucosal barrier may affect the transmission rates of HIV-1 infection in women.

Hormonal factors have been implicated in increasing a woman's risk of becoming infected with HIV-1, but the mechanisms involved in this increased susceptibility remain controversial (Quinn et al. 2000). However, the use of progesterone has been definitively proven to thin the vaginal epithelium of macaques, which is associated with markedly increased rates of vaginal SIV transmission. Recently, we have utilized this system to demonstrate the efficacy of candidate microbicide compounds in preventing intravaginal transmission of SHIV162P3 to macaques (Veazey et al. 2003a, 2003b, Lederman et al. 2004). Administering depo-provera (medroxyprogesterone acetate) to macaques approximately 4 weeks prior to vaginal inoculation with SIV or simian/human HIV results in marked vaginal epithelial thinning and infection of $\sim 90 \%$ animals (Marx et al. 1996, Veazey et al. 2003a). In addition, studies have demonstrated that the application of topical estrogen to macaques not receiving exogenous progesterone thickens the vaginal epithelium and reverses their susceptibility to infection (Smith et al. 2004). Thus, we and other researchers have hypothesized that hormonal changes that simply result in thinning or increased susceptibility to breaks in the vaginal epithelium may increase the susceptibility of women to vaginal transmission of HIV-1 and other sexually transmitted diseases (Marx et al. 1996, Veazey et al. 2003a, Smith et al. 2004, Quinn \& Overbaugh 2005).

Although it is presently debated, there is considerable evidence that hormones (progesterone) and/or thinning of the vaginal epithelium also increases the risk of HIV-1 transmission in women. For example, epidemiological evidence indicates that younger women are much more susceptible to HIV-1 infection (Quinn \& Overbaugh 2005). In addition, women who are pregnant or recently post partum have been shown to be more susceptible to HIV-1 transmission (Gray et al. 2005, Quinn \& Overbaugh 2005). These are the two conditions where progesterone is the dominant hormone, as estrogen levels are lower in premenopausal or pregnant women. Menses is also a period in which progesterone:estrogen ratios are increased, but determining whether women are more susceptible to HIV-1 or other infections during menses would be quite difficult. Moreover, interpreting such studies in women are highly complicated by the possibility that adolescent girls, women having intercourse during menses, or those who are pregnant may simply be more likely to engage in risky behavior (i.e. not use condoms). Thus, nonhuman primate models are necessary for assessing the susceptibility to vaginal HIV-1 transmission. 


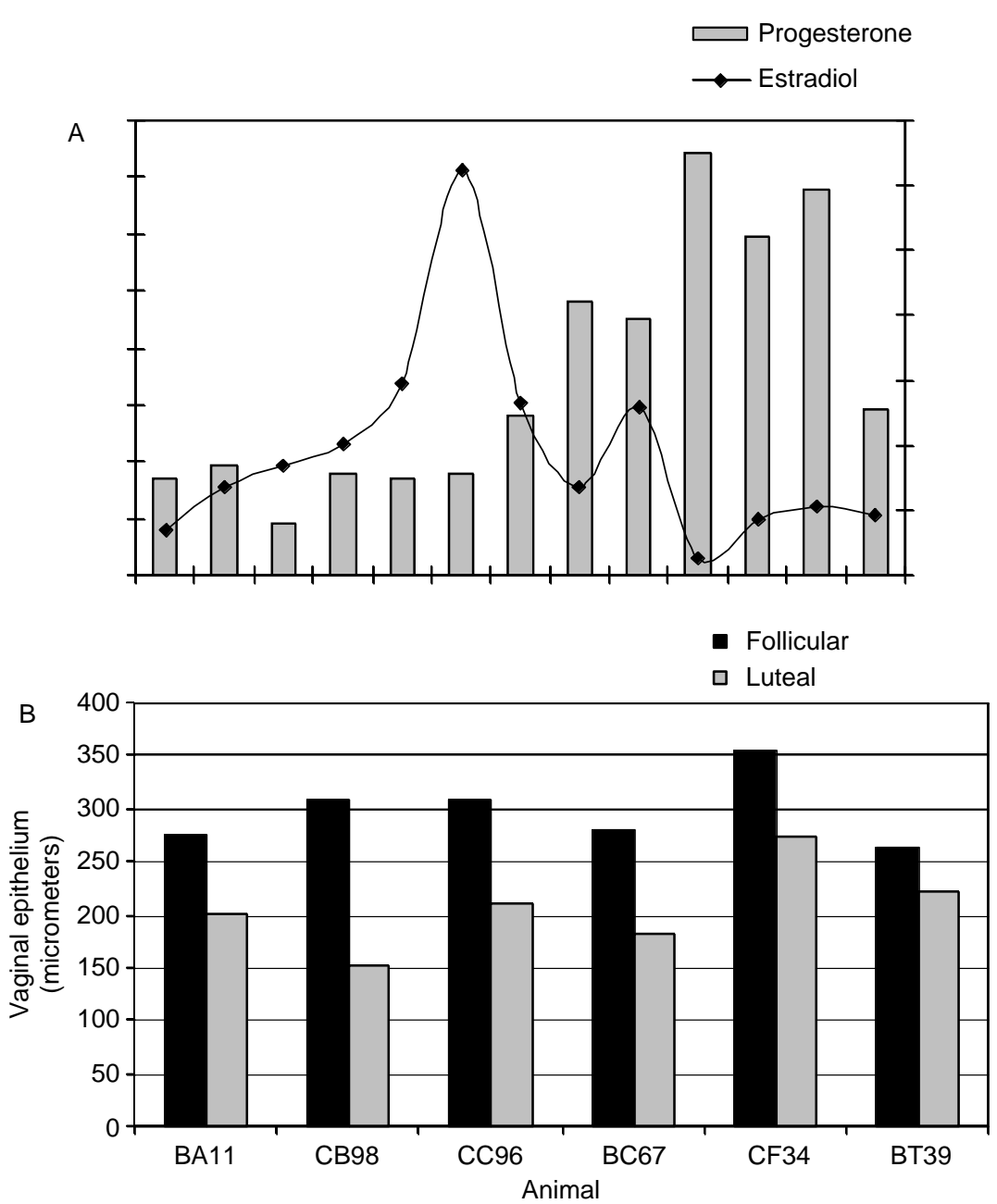

Figure 3 Cyclic changes in hormone levels in macaques followed through a menstrual cycle. (A) Average estrogen and progesterone levels in serum of macaques. Day 0 of the cycle was estimated by monitoring vaginal blood in these animals and graph represents 28 days from the first day of an observed menstrual cycle. Vaginal biopsies were collected at days 14 (follicular phase) and 28 (luteal phase). (B) Comparison of epithelial thickness of individual animals during luteal and follicular phases of menstrual cycle. The average vaginal epithelium was significantly thinner during luteal phase compared with follicular phase $(P<0 \cdot 01)$.

The studies here demonstrate that the vaginal epithelium of macaques undergoes significant alterations in thickness as the animal matures. The vaginal epithelium is thickest in the age group of 3-20 years, when female macaques are cycling and reproductively mature. Moreover, among this group, the vaginal epithelium is thickest and most keratinized in macaques during peak estrogen levels (follicular phase) and thinnest in luteal phase, when progesterone:estrogen ratios are the highest. We hypothesize that this reduced epithelial thickness during the luteal phase of menstrual cycle is responsible for the increased rates of transmission of SIV to macaques in this stage. However, few studies consider the stage of ovulation or menses when designing nonhuman primate studies of vaginal transmission. Unfortunately, animals in the aging study were housed in large outdoor breeding corrals making it impossible to accurately assess whether they were menstruating or postmenopausal. Moreover, ovarian samples were not collected at necropsy, and thus we could not address whether the thinning of the vaginal mucosa was associated with menopause (lack of ovulation) or declining responsiveness of the vagina to estrogen. However, regardless of the mechanism, these studies demonstrate that there is a clear thinning of the vaginal mucosa in elderly macaques suggesting they may be more susceptible to certain sexually transmitted diseases.

Recently, new models for testing microbicides are emerging in efforts to identify compounds that can be vaginally applied to reduce a woman's susceptibility to HIV-1 transmission. Again, however, either large numbers of animals or high doses of virus are required to generate significant results in non-hormone-treated macaques. To avoid using 

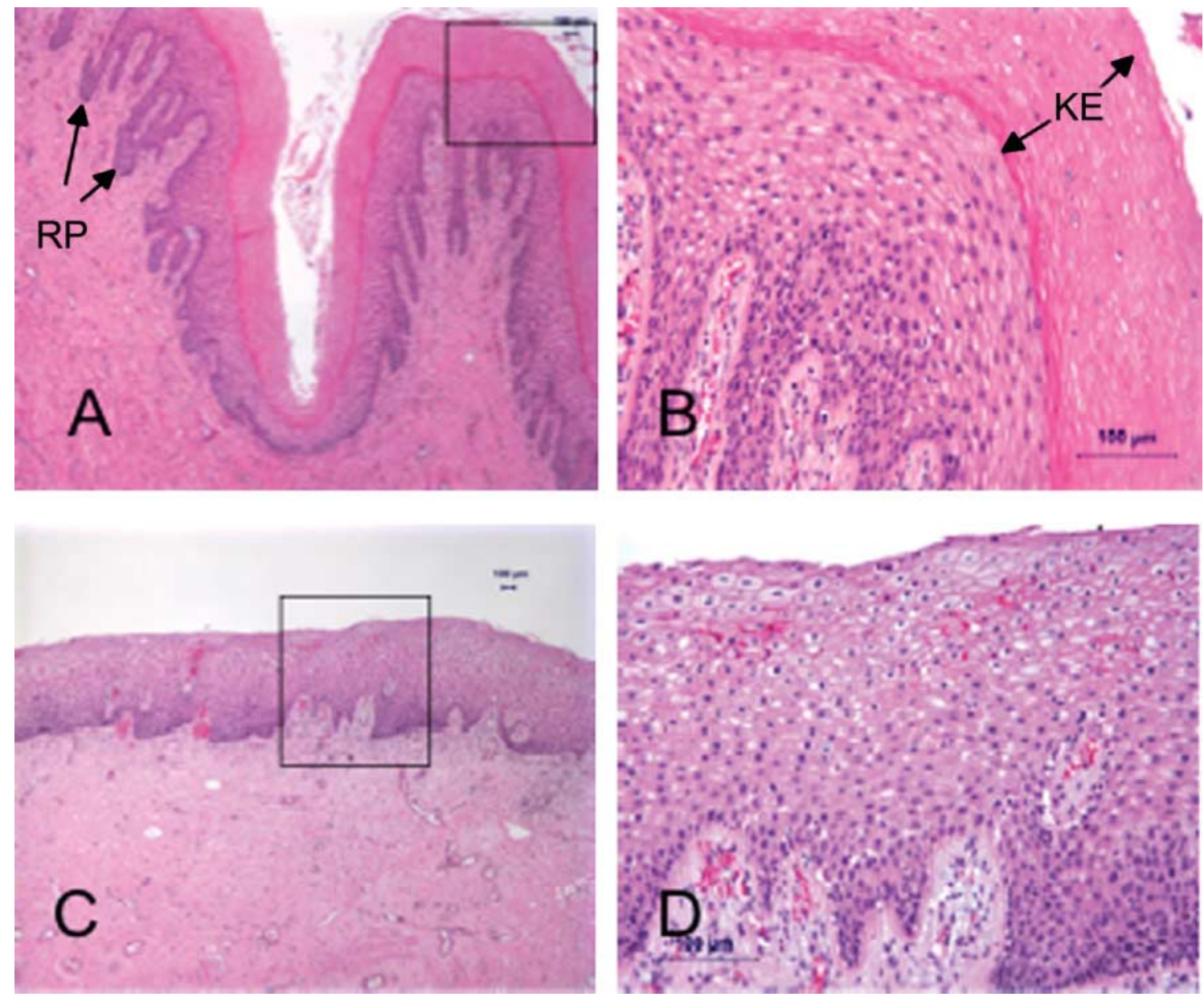

Figure 4 Variations in vaginal epithelial thickness and keratinization associated with changes in the menstrual cycle. (A) and (B) are representative of the vaginal mucosa in peak follicular phase (estrogen dominance), whereas (C) and (D) are from the same macaque in the luteal phase (menses, progesterone dominance). (B) and (D) are higher magnifications of regions of $(A)$ and $(C)$ respectively, as indicated by the box. Note that the vaginal epithelium is thick with a significant zone of keratinized epithelium (KE) and deep rete pegs (RP) in the follicular phase ((A) and (B)). In contrast, the mucosa lacks keratinization and deep rete pegs in the luteal phase $((C)$ and $(D))$. Biopsies were taken from the same macaque 14 days apart. (A) and (C) magnification X 100; (B) and (D) magnification X 200.

excessive amounts of virus, some propose using low doses of virus applied weekly to the vagina of macaques in an attempt to more closely mimic the 'natural' exposure of women to HIV-infected semen (Regoes et al. 2005). In these models, animals are repeatedly challenged weekly with low doses of virus until all control animals become infected (Otten et al. 2005, Regoes et al. 2005). However, multiple low-dose challenge models rely on the assumption that each exposure is identical, which may not be accurate. Based on the findings reported here, macaques may be much more susceptible to vaginal transmission during menses, a fact that might confuse such models if, for example, some macaques are dosed during menses and others during the follicular phase of the cycle.

Similar changes in the vaginal epithelium of women have been shown to occur at different stages of menses. Proliferation and maturation of vaginal epithelial cells in the vaginal mucosa during ovulation is well documented in women (Sjoberg et al. 1988). Since females are more likely to have intercourse during ovulation (peak follicular phase) increasing the layers and integrity of the vaginal epithelium is likely a physiologic mechanism to prepare the vaginal mucosa for the potentially traumatic effects of intercourse, as well as to the exposure of allogenic and/or environmental antigens that will be introduced into the vagina during intercourse. Squamous epithelial cells, particularly when several layers thick, are likely to present a formidable barrier to the entry of viruses and other potential pathogens into the vagina. This may be why vaginal estrogen therapy is beneficial in preventing or ameliorating vaginitis in postmenopausal women (Mainini et al. 2005). This may also explain why large doses of SIV are required to vaginally infect macaques without exogenous progesterone treatment. However, careful 
immunologic assessment of other factors that could play a role in HIV-1 transmission remain to be fully explored.

If the hypothesis that women who frequently have intercourse during menses, pregnancy, or specific age groups (juveniles) are more susceptible to HIV-1 is correct, it is possible that this could be affecting rates of transmission in specific populations. For example, in areas where economic or religious factors encourage women to use the 'rhythm method' (avoiding intercourse during the follicular phase), this could lead to increased rates of transmission. Regardless, developing effective prophylactic control measures against vaginal HIV transmission requires a better understanding of the potential factors influencing such transmission. Thus, a better knowledge about the physiologic, anatomic, and immunologic alterations that occur in the vaginal epithelium could help to design more effective prevention strategies.

\section{Acknowledgements}

We thank J LeBlanc, L Green, M Dodd, K Rasmussen, M Duplantis, and M Martin for technical assistance.

\section{Funding}

This work was supported by NIH/NIAID grants AI65413, AI51649, AI062410, and NCRR RR00164. The authors declare that there is no conflict of interest that would prejudice the impartiality of this scientific work.

\section{References}

Gray RH, Li X, Kigozi G, Serwadda D, Brahmbhatt H, Wabwire-Mangen F, Nalugoda F, Kiddugavu M, Sewankambo N, Quinn TC et al. 2005 Increased risk of incident HIV during pregnancy in Rakai, Uganda: a prospective study. Lancet 366 1182-1188.

Lederman MM, Veazey RS, Offord R, Mosier DE, Dufour J, Mefford M, Piatak M Jr., Lifson JD, Salkowitz JR, Rodriguez B et al. 2004 Prevention of vaginal SHIV transmission in rhesus macaques through inhibition of CCR5. Science 306 485-487.

Ma Z, Lu FX, Torten M \& Miller CJ 2001 The number and distribution of immune cells in the cervicovaginal mucosa remain constant throughout the menstrual cycle of rhesus macaques. Clinical Immunology 100 240-249.

Mainini G, Scaffa C, Rotondi M, Messalli EM, Quirino L \& Ragucci A 2005 Local estrogen replacement therapy in postmenopausal atrophic vaginitis: efficacy and safety of low dose 17 beta-estradiol vaginal tablets. Clinical and Experimental Obstetrics and Gynecology 32 111-113.

Marx PA, Compans RW, Gettie A, Staas JK, Gilley RM, Mulligan MJ, Yamschikov GV, Chen D \& Eldridge JH 1993 Protection against vaginal SIV transmission with microencapsulated vaccine. Science $\mathbf{2 6 0}$ $1323-1327$.
Marx PA, Spira AI, Gettie A, Dailey PJ, Veazey RS, Lackner AA, Mahoney CJ, Miller CJ, Claypool LE, Ho DD et al. 1996 Progesterone implants enhance SIV vaginal transmission and early virus load. Nature Medicine 2 1084-1089.

Miller CJ, Alexander NJ, Sutjipto S, Lackner AA, Gettie A, Hendrickx AG, Lowenstine LJ, Jennings M \& Marx PA 1989 Genital mucosal transmission of simian immunodeficiency virus: animal model for heterosexual transmission of human immunodeficiency virus. Journal of Virology 63 4277-4284.

Miller CJ, Alexander NJ, Vogel P, Anderson J \& Marx PA 1992 Mechanism of genital transmission of SIV: a hypothesis based on transmission studies and the location of SIV in the genital tract of chronically infected female rhesus macaques. Journal of Medical Primatology 21 64-68.

Otten RA, Adams DR, Kim CN, Jackson E, Pullium JK, Lee K, Grohskopf LA, Monsour M, Butera S \& Folks TM 2005 Multiple vaginal exposures to low doses of R 5 simian-human immunodeficiency virus: strategy to study HIV preclinical interventions in nonhuman primates. Journal of Infectious Diseases 191 164-173.

Quinn TC \& Overbaugh J 2005 HIV/AIDS in women: an expanding epidemic. Science 308 1582-1583.

Quinn TC, Wawer MJ, Sewankambo N, Serwadda D, Li C, WabwireMangen F, Meehan MO, Lutalo T \& Gray RH 2000 Viral load and heterosexual transmission of human immunodeficiency virus type 1. Rakai Project Study Group. New England Journal of Medicine 342 921-929.

Regoes RR, Longini IM, Feinberg MB \& Staprans SI 2005 Preclinical assessment of HIV vaccines and microbicides by repeated low-dose virus challenges. PLoS Medicine 2 e249.

Shattock RJ \& Moore JP 2003 Inhibiting sexual transmission of HIV-1 infection. Nature Reviews Microbiology $125-34$.

Sjoberg I, Cajander S \& Rylander E 1988 Morphometric characteristics of the vaginal epithelium during the menstrual cycle. Gynecologic and Obstetric Investigation 26 136-144.

Smith SM, Mefford M, Sodora D, Klase Z, Singh M, Alexander N, Hess D \& Marx PA 2004 Topical estrogen protects against SIV vaginal transmission without evidence of systemic effect. Acquired Immune Deficiency Syndrome 18 1637-1643.

Sodora DL, Gettie A, Miller CJ \& Marx PA 1998 Vaginal transmission of SIV: assessing infectivity and hormonal influences in macaques inoculated with cell-free and cell-associated viral stocks. AIDS Research and Human Retroviruses 1 S119-S123.

Veazey RS, Shattock RJ, Pope M, Kirijan JC, Jones J, Hu Q, Ketas T, Marx PA, Klaase PJ, Burton DR et al. 2003a Prevention of virus transmission to macaque monkeys by a vaginally applied monoclonal antibody to HIV gp120. Nature Medicine 9 343-346.

Veazey RS, Klasse PJ, Ketas TJ, Reeves JD, Piatak M, Jr., Kunstman K, Kuhmann SE, Marx PA, Lifson JD, Dufour J et al. $2003 b$ Use of a small molecule CCR 5 inhibitor in macaques to treat simian immunodeficiency virus infection or prevent simian-human immunodeficiency virus infection. Journal of Experimental Medicine 198 1551-1562.

Received in final form 16 April 2006

Accepted 16 May 2006

Made available online as an Accepted Preprint 12 June 2006 